\title{
A novel strategy for tailoring cerium oxide polyaniline nanocomposites for photocatalytic preparation of aniline
}

\author{
Mohammad W Kadi ${ }^{1}$ and Reda M Mohamed ${ }^{1,2 *}$ \\ ${ }^{1}$ Department of Chemistry, Faculty of Science, King Abdulaziz University, P.O. Box 80203, Jeddah 21589, Kingdom of Saudi Arabia \\ ${ }^{2}$ Advanced Materials Department, Central Metallurgical R\&D Institute, CMRDI, P.O. Box 87, Helwan, Cairo 11421, Egypt
}

\begin{abstract}
Cerium oxide polyaniline nanocomposites were synthesized in an effective manner to produce a uniform core-shell structure of the particles. This nanocomposite was then used in the photocatalytic synthesis of aniline. Synthesis conditions, heat treatment, and other factors that affect the structural properties of nanocomposite were studied extensively to optimize the synthesis process. Polyaniline nanospheres (PANI_NS) were prepared by the microemulsion method. Polyaniline nanoparticles (PANI_NP) were fabricated through polymerization (chemical oxidative process) of aniline. Specific surface area values of PANI_NS, CeO_@PANI_NS, PANI_ NP, CeO $@$ @PANI_NP, and $\mathrm{CeO}_{2} \_$NS were 200, 260, 40, 110 and $130 \mathrm{~m}^{2} / \mathrm{g}$ respectively. The photo-catalytic effeciency of the nanocomposite was investigated through converting nitrobenzene to aniline via reduction reaction under visible light illumination. CeO @PANI_NS outperforms CeO $@$ @ANI_NP 1.4 times, PANI_NS 2.5 times, PANI_NP 6.6 times, $\mathrm{CeO}_{2} \_$NS 14.3 times in terms of photocatalytic reduction of nitrobenzene to aniline.
\end{abstract}

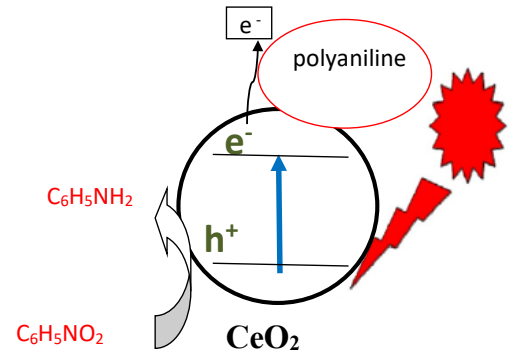

\section{Introduction}

Polyaniline (PANI) is a conjugated polymer that attracted researcher's attention as it possesses favourable properties towards photocatalysis and other applications. These properties include absorption of light in the visible range, environmental stability, high conductivity, and corrosion resistance [1]. Generally, inorganic nano oxides such as $\mathrm{TiO} 2, \mathrm{ZnO}, \mathrm{CeO}_{2}, \mathrm{WO}^{-3}$, and many others; have been widely used in photocatalytic reactions. Researchers also try to combine inorganic nanomaterials with organic polymers in efforts to prepare catalytic composites with high efficiency. Ameen, et al. synthesized a PANI $\mathrm{ZnO}$ composite and used it effectively in methylene blue dye degradation [2]. Sharma, et al. synthesized PANI/CdS nanoflowers and nanorods nanocomposites and used them in photodegradation [3]. Ozbay, et al. reported photo-catalytic efficiencies of polyanilinemodified $\mathrm{ZnO}$ and $\mathrm{TiO}_{2}$ beneath Vis light [1]. The photocatalytic efficiency of polyaniline sensitized nano-crystallite titania structures has been investigated by Min, et al. [4]. Many researchers studied the various structure of $\mathrm{PANI}$ and $\mathrm{TiO}_{2}$ composites. These structures include nanotubes [5,6], bionic nanopapilla [7], and A core-shell structure [8]. Agarwal reported the fabrication of polyaniline $/ \mathrm{ZrO}_{2}$ oxide conductive nano-composite required for many applications such as adsorption of dyes [9]. Patil, et al. developed a sensor for the detection of $\mathrm{NH}_{3}$ utilizing copper nanoparticle intercalated polyaniline nanocomposite thin films [10]. Salvatierra, et al. studied Carbon nanotube/polyaniline nanocomposites [11].

$\mathrm{CeO}_{2}$ is known to be a good catalyst for its special redox property and high oxygen storage capacity this catalyst has been studied by numerous researchers [12-14]. PANI and $\mathrm{CeO}_{2}$ combination also received some attention. Yongjun $\mathrm{He}$ reported the synthesis of polyaniline/nano $\mathrm{CeO}_{2}$ arrangement microspheres by means of a solid-stabilized emulsion regime. The synthesized Polyaniline/nano$\mathrm{CeO}_{2}$ composite microspheres had an average diameter of $7 \mathrm{~mm}$ [15]. Chuang and Yang used $\mathrm{CeO}_{2}$ to oxidize aniline for the fabrication of $\left(\mathrm{CeO}_{2} / \mathrm{PANI}\right)$ core-shell nano structures. They reported a polygonal shape of the PANI/ $\mathrm{CeO}_{2}$ nanocomposites [16]. Kumar, et al. exploited in-situ polymerization to prepare the $\mathrm{PANI} / \mathrm{CeO}_{2}$ nanocomposite and reported the structure of this composite [17]. Sasikumar, et al. reported the use of aniline, $\mathrm{HCl}, \mathrm{CeO}_{2}$ nanoparticles, ammonium persulfate, and

${ }^{\star}$ Correspondence to: Mohamed RM, Department of Chemistry, Faculty of Science, King Abdulaziz University, P.O. Box 80203, Jeddah 21589, Kingdom of Saudi Arabia, E-mail: redama123@yahoo.com

Key words: $\mathrm{CeO}_{2}$, polyaniline, nanospheres, nanoparticles, photocatalytic preparation of aniline, visible light

Received: July 05, 2019; Accepted: July 27, 2019; Published: July 31, 2019 
water to synthesize $\mathrm{PANI} / \mathrm{CeO}_{2}$ composite and used it as a corrosion inhibitor [18]. Da Silva, et al. reported the fabrication of poly-aniline ES-PANI Cl-salt in the existence of $\mathrm{CeO}_{2}$. They concluded that $\mathrm{CeO}_{2}$ molecules designing a core-shell arrangement with non-homogeneous shape and size have been coated with a delicate layer of ES-PANI nanotissues [19].

Here we report, a novel method for the preparation of a uniform core-shell spherical PANI $\mathrm{CeO}_{2}$ nanocomposite. In the preparation of PANI_NS, ammonium persulfate was used as an oxidant, ethanol and n-butyl alcohol was utilized as solvents, Na-salt of dodecyl benzene sulfonic acid was used as a surfactant. Poly vinyl pyrrolidine was used in the preparation of $\mathrm{CeO}_{2} @ \mathrm{PANI}$ nanocomposites.

\section{Chemicals and experimental procedures}

\section{Chemicals}

Monomers of aniline (purity of $99 \%$, Aldrich), cerium isopropoxide, poly vinyl pyrrolidine (PVP), ammonium persulfate (APS), sodium dodecylbenzene sulfonate (SDBS), ethanol (99.8\%), n-butyl alcohol (BA), and hydrochloric acid ( $\mathrm{HCl}, 37 \%)$ have been utilized without any further purification.

\section{Fabrication of polyaniline nanospheres}

The microemulsion method was employed to prepare polyaniline nanospheres (PANI_NS). In this procedure, $6.0 \mathrm{~g}$ of sodium dodecyl benzene sulfonate (SDBS) was dissolved in $100 \mathrm{~mL}$ of distilled water while maintaining the $\mathrm{pH}=0.3$ with the use of $\mathrm{HCl}$ solution. $1.3 \mathrm{~mL}$ of aniline monomer was added to the mixture. After stirring for $60 \mathrm{~min} 4$ $\mathrm{mL}$ of n-butyl alcohol (BA) was drop-wisely introduced to the mixture. The system temperature was adjusted at $25{ }^{\circ} \mathrm{C}$, and $33 \mathrm{~mL}$ of $10 \%$ ammonium persulfate (APS) solution was introduced to the reaction medium and the resultant system was agitated for additional $5 \mathrm{~h}$ to achieve polymerization of the aniline monomer. A change of colour from brown-yellow to dark-green was observed during the stirring process. The mixture was cleaned several times via ethanol and distilled water and then left to dry for $12 \mathrm{~h}$ at $70^{\circ} \mathrm{C}$ using a vacuum oven to collect PANI_NS.

\section{Preparation of polyaniline nanoparticles}

Polymerization of aniline was performed to fabricate poly-aniline nanoparticles (PANI_NP) adopting the chemical oxidation route. In this procedure, $1.3 \mathrm{~mL}$ of aniline monomer was added to $100 \mathrm{~mL}$ of $\mathrm{HCl}$ aqueous solution under vigorous stirring at $1^{\circ} \mathrm{C}$. A $33 \mathrm{~mL}$ portion $10 \%$ APS solution was introduced to the system which was agitated for $5 \mathrm{~h}$. The mixture was cleaned many intervals with ethyl alcohol and distilled water under vacuum. The resultant yield was kept to dry for 30 $\mathrm{h}$ at $70^{\circ} \mathrm{C}$ using a vacuum oven and PANI_NP for further use.

\section{Preparation of $\mathrm{CeO}_{2} @ \mathrm{PANI}$ nanocomposites}

A mixture of $0.3 \mathrm{~g}$ of PVP and $1.6 \mathrm{~g}$ of PANI_ NS or PANI_NP were dispersed in $30 \mathrm{~mL}$ of ethanol using Ultrasonic Cleaner for 60 $\mathrm{min}$. Then, cerium isopropoxide solution $(100 \mathrm{~mL}$ of $0.3 \mathrm{~mol} / \mathrm{L})$ was added to the mixture with further dispersion in the ultrasonic bath for $3 \mathrm{hrs}$. The mixture was cleaned many intervals with distilled water and ethanol under vacuum distillation. After drying overnight at $100^{\circ} \mathrm{C}$, the product material was calcined for $4 \mathrm{~h}$ at $350^{\circ} \mathrm{C}$. Both $\mathrm{CeO}_{2} @$ PANI_NS and $\mathrm{CeO}_{2} @$ PANI_NP were synthesized by this mannar. For comparison purposes, pure $\mathrm{CeO}_{2}$ nanospheres were prepared by the above-mentioned procedures without the use of PANI_NS or PANI_NP.

\section{Characterization}

JEOL-JEM-1230 transmission electron microscope was used to observe the morphology and sample dimensions of the prepared materials. To get the TEM images, samples were ultrasonicated for 30 $m$ after suspension in ethanol. A small portion of the suspended sample was left to dry on the carbon covered $\mathrm{Cu}$ grid and introduced to the spectrometer.

The surface area was determined via $\mathrm{N}_{2}$-adsorption evaluations of the specimen adopting Chromatech instrument (Nova 2000 series) at $77 \mathrm{~K}$. specimens were heated at $100^{\circ} \mathrm{C}$ beneath vacuum for $2 \mathrm{~h}$ to complete this measurement.

Bruker axis D8 XRD system was utilized to observe the crystallite points of the nanocomposites. XRD was observed employing $\mathrm{Cu} \mathrm{Ka}$ radiation $(\lambda=1.540 \AA)$ at ambient temperature.

A spectrometer (Thermo-Scientific K-ALPHA) has been utilized in order to attain x-ray photoelectron spectroscopy (XPS) measurements. Whereas, a spectrophotometer (Jasco, Japan) was adopted to attain UVVis-DRS spectra that were recognized at ambient temperature in the absorption space from 200 up to $800 \mathrm{~nm}$. Band gap performance has been determined by applying the observed UV-Vis-DRS. In addition, a fluorescence spectrophotometer of Shimadzu type has been utilized to record photoluminescence emission spectra (PL). A transmittance mode FTIR spectrum was documented for a powdered sample and $\mathrm{KBr}$ applying a JASCO-type spectrometer keeping the spectral resolution of $2 \mathrm{~cm}^{-1}$ at ambient temperature.

\section{Photo-catalytic tests}

The efficiency of the prepared nanocomposites was tested on the reduction of nitrobenzene into aniline. For this purpose, a known weight of the photocatalyst was scattered by means of ultrasonic in a $10 \mathrm{~mL}$ nitrobenzene- $\mathrm{CH} 3 \mathrm{OH}$ solution $(1 / 99, \mathrm{v} / \mathrm{v})$ with starting dose of nitrobenzene $(\mathrm{NB})$ at $8.13 \times 10^{-4} \mathrm{molL}^{-1}$. The reaction mixture was illuminated under artificial visible light produced from Xenon lamp mounted on a photocatalytic reactor. A $\lambda>420 \mathrm{~nm}$ cutoff filter was used and a running water tube was exploited to prevent heating which allowed the reaction solution to be kept at about $30^{\circ} \mathrm{C}$. The fixed container, made of quartz, was placed at $11 \mathrm{~cm}$ from the origin of illumination. Before starting, dissolute $\mathrm{O}_{2}$ is flushed from the solution by nitrogen for $0.5 \mathrm{~h}$. Illumination time was set at $2.5 \mathrm{~h}$ for each experiment. After exposure specimens were removed from the reactor, centrifuged for $20 \mathrm{~min}(7000 \mathrm{rpm})$. Finally, the solution was filtered via a filter of $0.2-$ $\mu \mathrm{m}$ pore size in order to get rid of any remaining molecules. Agilent Gas Chromatograph (GC 7890A model) was applied to analyze the resulting aniline from the reduction process.

\section{Results and discussion}

\section{Characterizations of materials}

Figure 1 shows XRD diffractograms of PANI_NP, PANI_NS, $\mathrm{CeO}_{2-}$ NS, CeO $@$ @PANI_NP, and $\mathrm{CeO}_{2} @ P A N I \_N S$ samples. The broad peak at $\sim 15-25^{\circ} \mathrm{C}$ suggests polyaniline phase structure for PANI_NP, PANI NS. On the other hand, $\mathrm{CeO}_{2}$ NS, CeO $@$ @PANI_NP, and CeO $@$ @PANI_ NS are composed of $\mathrm{CeO}_{2}$ phase as characteristic peaks of polyaniline disappeared in the spectra of $\mathrm{CeO}_{2} @ P A N I \_N P$ and $\mathrm{CeO}_{2} @ P A N I \_N S$. Also, the decrease in the intensity of the characteristic peaks of $\mathrm{CeO}_{2}$ phase in the spectra of $\mathrm{CeO}_{2} @ P A N I \_N P$ and $\mathrm{CeO}_{2} @ P A N I \_N S$ suggests that doping of polyaniline decrease crystallite sizes of both composites. 


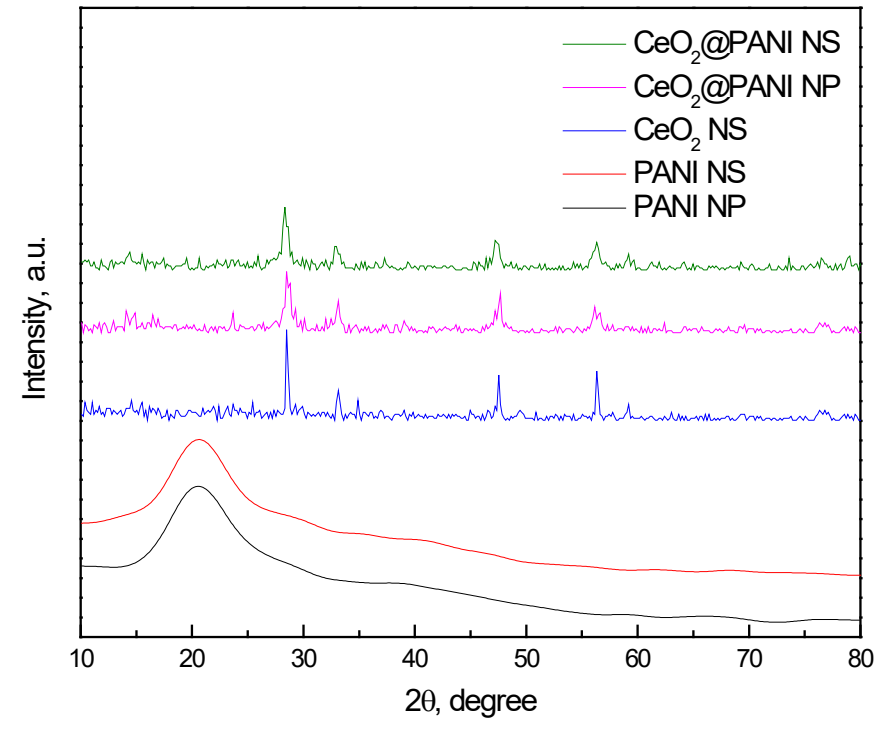

Figure 1. XRD patterns of PANI_NP, PANI_NS, $\mathrm{CeO}_{2} \mathrm{NS}, \mathrm{CeO}_{2} @$ PANI_NP, and $\mathrm{CeO}_{2} @$ PANI_NS

Figure 2 exhibits TEM pictures of PANI_NP, PANI_NS, $\mathrm{CeO}_{2-}$ NS, CeO $@$ PANI_NP, and $\mathrm{CeO}_{2} @ P A N I \_N S$ samples. PANI_NP and CeO $@$ PANI_NP are nanoparticle shape with sizes of 60 and $75 \mathrm{~nm}$, respectively. However, $\mathrm{CeO}_{2}$ NS, PANI_NS, and $\mathrm{CeO}_{2} @ P A N I \_N S$ are nanospherical in shape with shell diameters of 20-40, 22-44 and 25-50 $\mathrm{nm}$, respectively and core diameter of 150,170 and $190 \mathrm{~nm}$, respectively. It is obvious that the addition of polyaniline increases the size of $\mathrm{CeO}_{2} @$ PANI_NS particles.

XPS spectra of Ce3d (A), O1s (B), N 1s (C) and C 1s (D) for CeO $@$ PANI_NS sample is illustrated in Figure 3 The existence of double binding peaks for $\mathrm{Ce} 3 \mathrm{~d} 5 / 2$ and $\mathrm{Ce} 3 \mathrm{~d} 5 / 2$ at 882 and $916 \mathrm{eV}$ indicate that the cerium ion is $\mathrm{Ce}(\mathrm{IV})$ (Figure $3 \mathrm{~A}$ ). The presence of one binding peak for $\mathrm{O} 1 \mathrm{~s}$ at $531.6 \mathrm{eV}$ (Figure 3B) indicates that oxygen ion was present as $\mathrm{O}^{2-}$. Therefore, The XPS results for $\mathrm{O} 1 \mathrm{~s}$ and Ce3d confirm the presence of $\mathrm{CeO}_{2}$. XPS spectra for $\mathrm{N} 1 \mathrm{~s}$ and $\mathrm{C} 1 \mathrm{~s}$ assure the existence of aniline in $\mathrm{CeO}_{2} @$ PANI_NS sample, due to the presence of the binding peaks for $\mathrm{N} 1 \mathrm{~s}$ and $\mathrm{C} 1 \mathrm{~s}$ at 400 and $284.7 \mathrm{eV}$ (Figures 3C and D, respectively).

FT-IR spectral lines for PANI_NS and $\mathrm{CeO}_{2} @ P A N I \_N S$ samples are exhibited in Figure 4. The characteristic peaks at 1567, 1492, 1297, 1245, 1147 and $815 \mathrm{~cm}^{-1}$ for polyaniline are present in PANI_NS and $\mathrm{CeO}_{2} @$ PANI_NS samples, which confirm the presence of polyaniline in $\mathrm{CeO}_{2} @ P A N I \_N S$ samples.

Figure 5 exhibits adsorption-desorption isotherms of $\mathrm{CeO}_{2-} \mathrm{NS}$, $\mathrm{CeO}_{2} @ P A N I \_N P$, and $\mathrm{CeO}_{2} @ P A N I \_N S$ respectively. Type II isotherm is present for PANI_NP sample as shown in Figure 5B. While type IV isotherm was present for $\mathrm{CeO}_{2-} \mathrm{NS}$ and $\mathrm{CeO}_{2} @ P A N I \_N S$ samples as shown in Figure 5. Therefore, $\mathrm{CeO}_{2-} \mathrm{NS}$ and $\mathrm{CeO}_{2} @ P A N I \_N S$ samples are mesoporous materials.

The pore size distribution of $\mathrm{CeO}_{2} @$ PANI_NS specimen is illustrated in Figure 6. A narrow distribution of pore size around 3.5 is obvious. This explains that the core-shell structure has a large surface area. The values of the specific surface area of PANI_NS, $\mathrm{CeO}_{2} @$ PANI_NS, PANI_NP, CeO $2_{2} @ P A N I \_N P$ and $\mathrm{CeO}_{2}$ NS as calculated from desorption measurements are 200, 260, 40, 110 and $130 \mathrm{~m}^{2} / \mathrm{g}$, respectively.
Figure 7 shows UV-Vis spectra of $\mathrm{CeO}_{2} \mathrm{NS}, \mathrm{CeO}_{2} @ P A N I \_N P$, and $\mathrm{CeO}_{2} @$ PANI_NS samples. The curve shows that $\mathrm{CeO}_{2} \mathrm{NS}$ absorbs in the UV zone, the existence of polyaniline within the nanoparticles and within the nanospheres results in a displacement of the absorption edge of cerium dioxide in the UV visible zone. Furthermore, the shift is greater in the case of polyaniline nanospheres. The values of band gap energies as calculated from UV-Vis spectra of $\mathrm{CeO}_{2} \mathrm{NS}, \mathrm{CeO}_{2} @ \mathrm{PANI}$ NP, and $\mathrm{CeO}_{2} @$ PANI_NS, are 3.21, 2.38 and $1.91 \mathrm{eV}$, respectively.

Figure 8 show $\mathrm{Pl}$ spectra of $\mathrm{CeO}_{2-} \mathrm{NS}, \mathrm{CeO}_{2} @ P A N I \_\mathrm{NP}$ and $\mathrm{CeO}_{2} @$ PANI_NS samples. $\mathrm{CeO}_{2}$ NS shows high $\mathrm{Pl}$ peak intensity, the addition of polyaniline to the nanoparticles and nanospheres decreases the $\mathrm{Pl}$ peak intensity. Furthermore, the decrease in the case of polyaniline nanospheres is greater than that of polyaniline nanoparticles. The values of band gap energies of $\mathrm{CeO}_{2}-\mathrm{NS}, \mathrm{CeO}_{2} @ P A N I \_N P$ and $\mathrm{CeO}_{2} @$ PANI_NS samples as determined from their $\mathrm{Pl}$ emission spectra are $3.20,2.37$ and $1.90 \mathrm{eV}$, respectively. These values are very close to those obtained from UV-Vis spectra as discussed in the previous paragraph.

\section{Photocatalytic performance}

Conversion of nitrobenzene into aniline reaction was utilized to test the synthesized photocatalyst. A comparison between various prepared

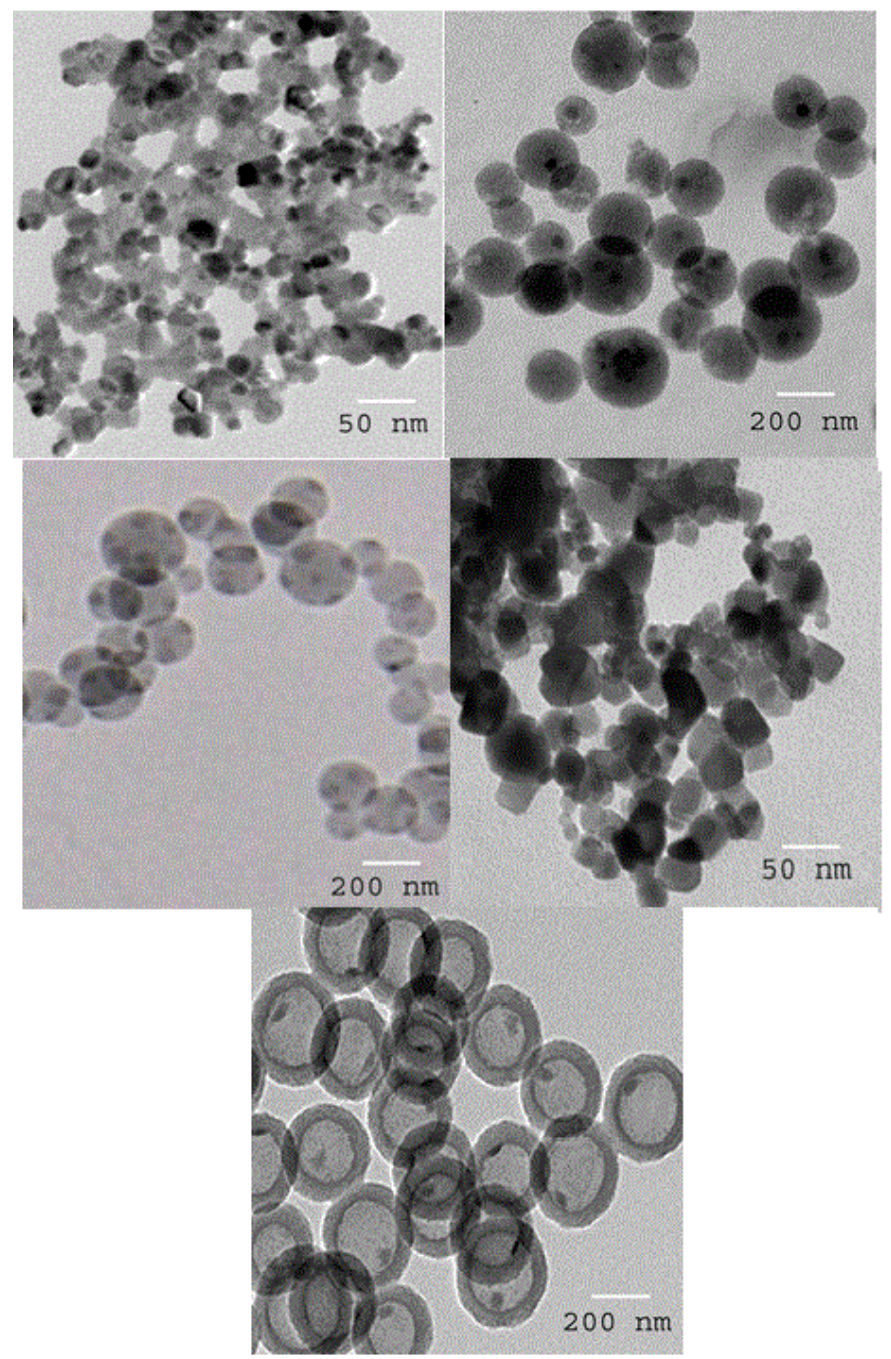

Figure 2. TEM images of PANI_NP(A), PANI_NS (B), $\mathrm{CeO}_{2} \mathrm{NS}(\mathrm{C}), \mathrm{CeO}_{2} @$ PANI_NP (D) and $\mathrm{CeO}_{2} @$ PANI_NS (E) 

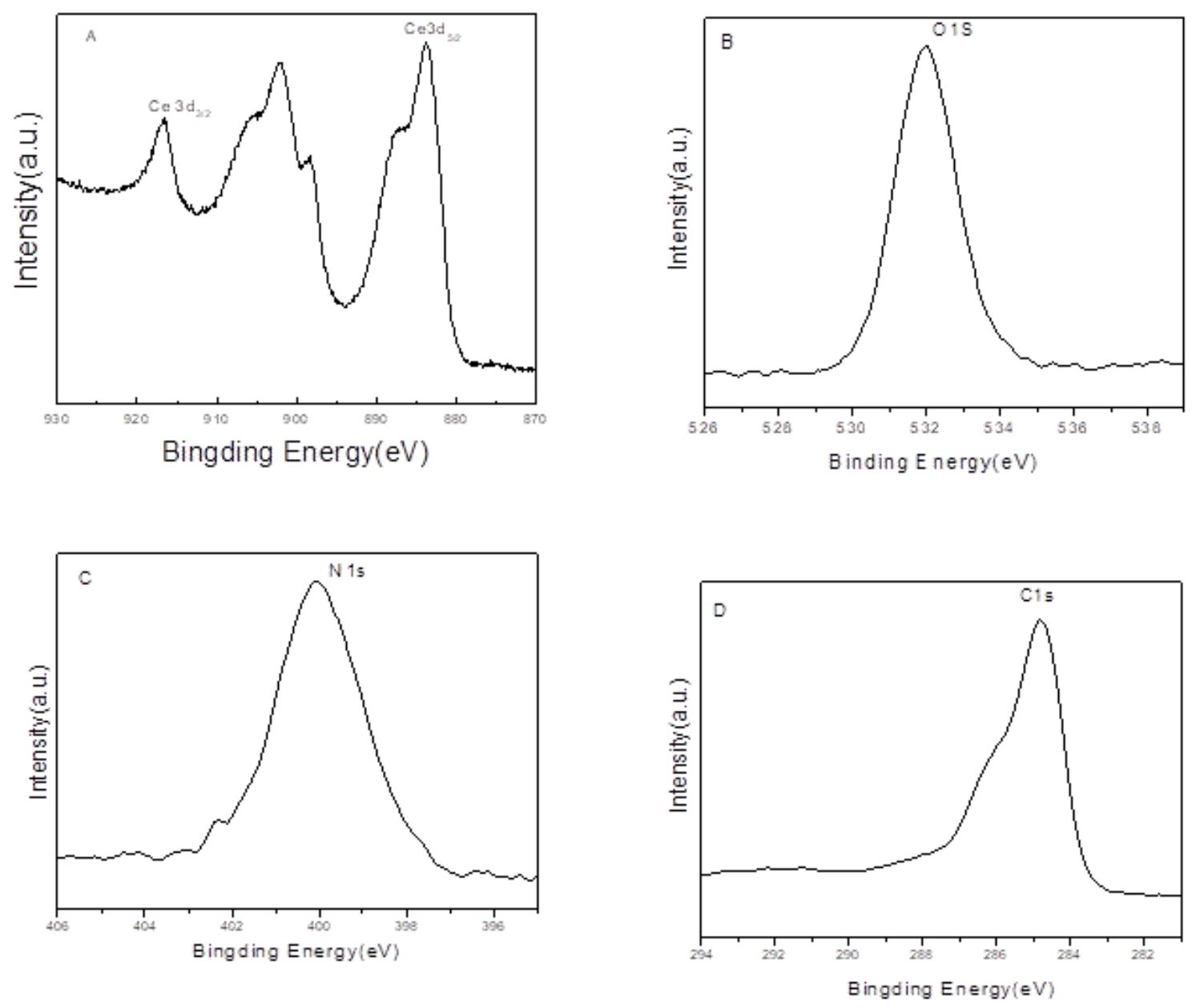

Figure 3. XPS spectra of Ce3d (A), O1s (B), N1s (C) and C1s (D) for $\mathrm{CeO}_{2} @$ PANI_NS sample

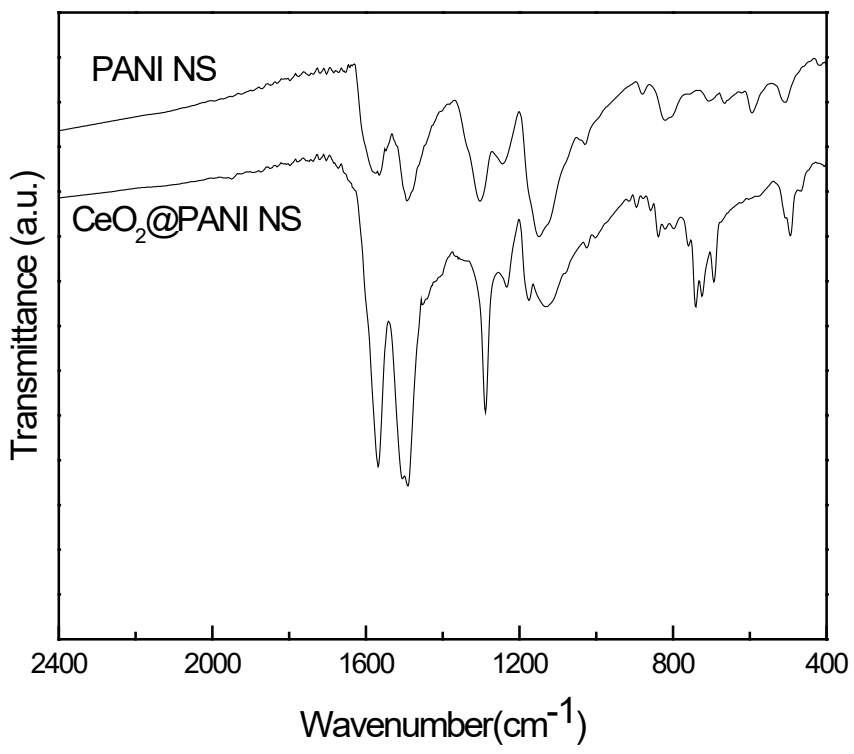

Figure 4. FT-IR spectra for PANI_NS and $\mathrm{CeO}_{2} @$ PANI_NS 

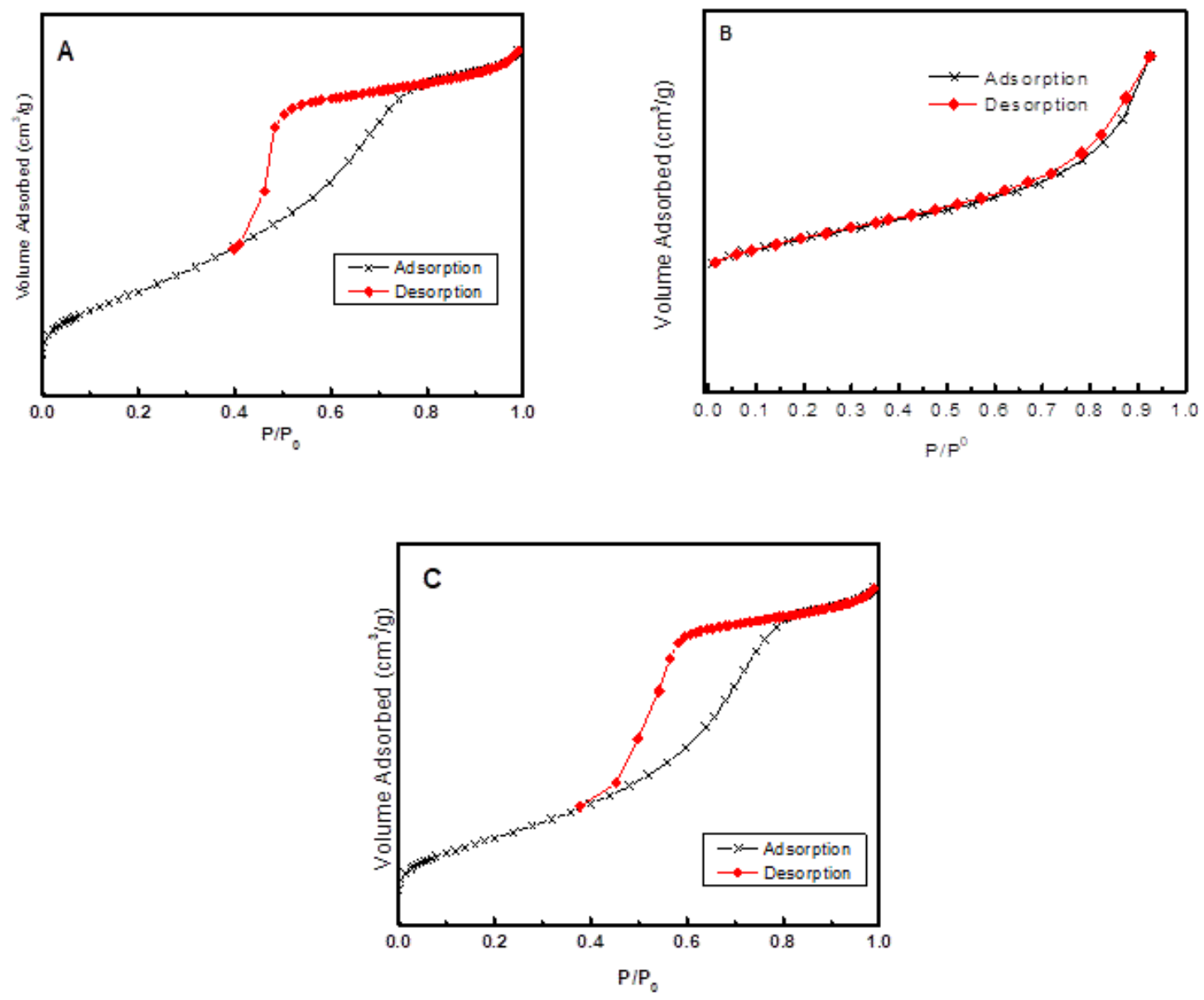

Figure 5. Adsorption-desorption isotherms of $\mathrm{CeO}_{2} \mathrm{NS}(\mathrm{A}), \mathrm{CeO}_{2} @ \mathrm{PANI}$-NP (B) and $\mathrm{CeO}_{2} @ \mathrm{PANI}$-NS (C)

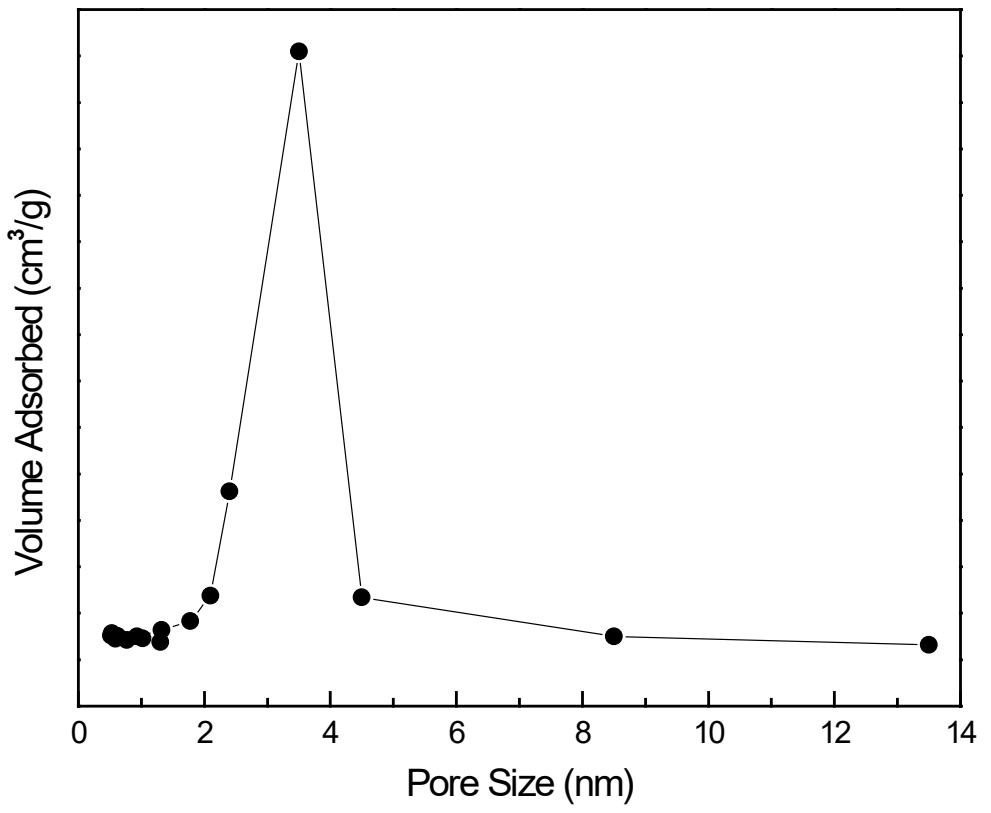

Figure 6. Pore size distribution of $\mathrm{CeO}_{2} @$ PANI_NS sample 


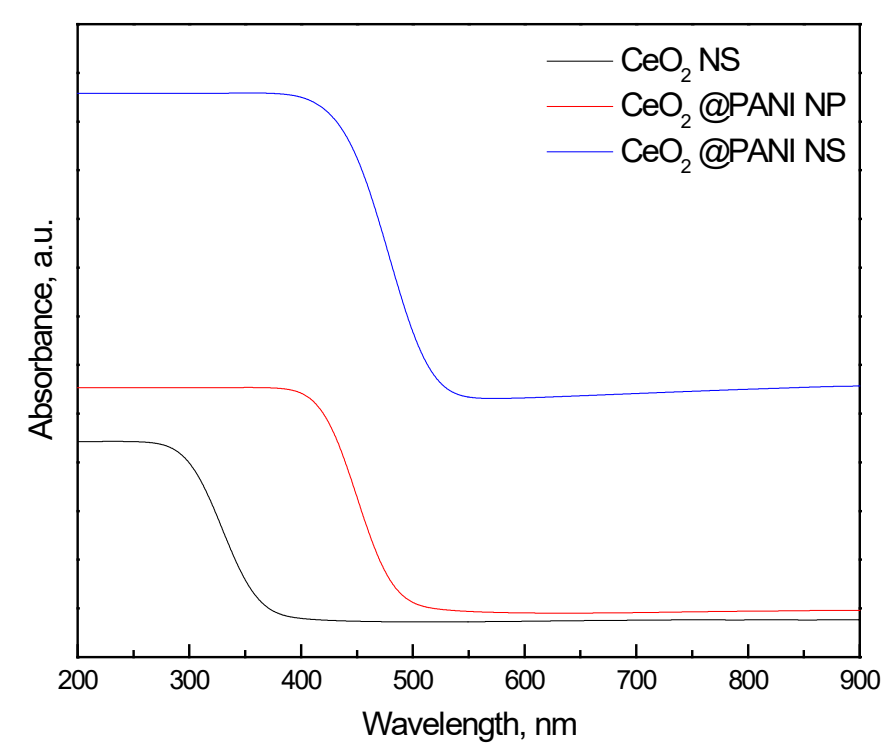

Figure 7. UV-Vis spectra of $\mathrm{CeO}_{2} \mathrm{NS}_{-}, \mathrm{CeO}_{2} @ \mathrm{PANI} \_\mathrm{NP}$, and $\mathrm{CeO}_{2} @$ PANI_NS samples

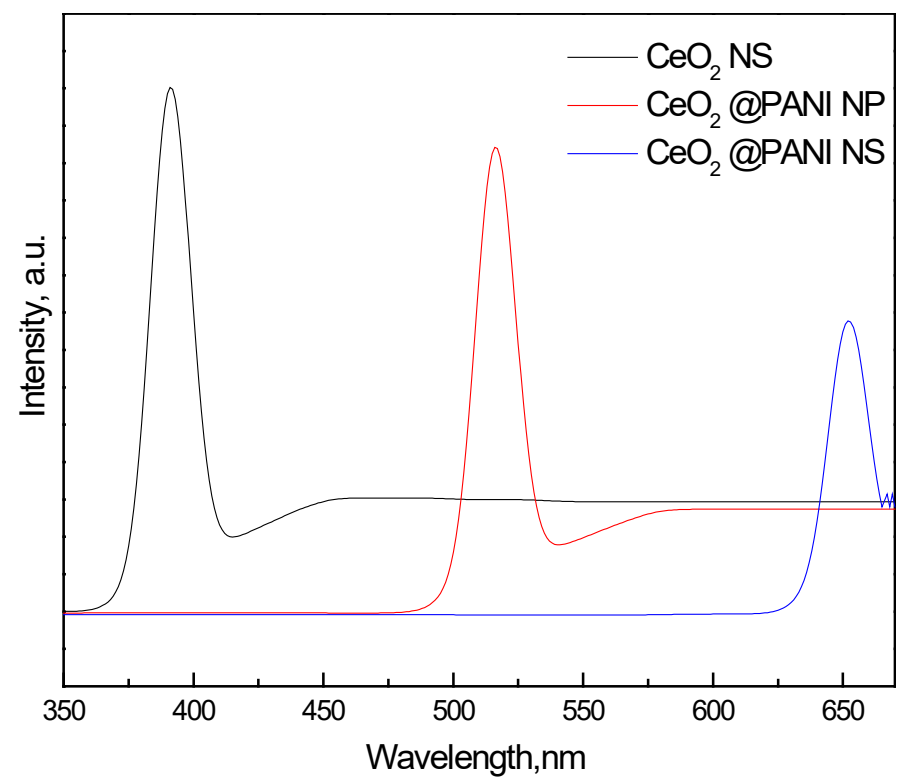

Figure 8. $\mathrm{Pl}$ spectra of $\mathrm{CeO}_{2} \mathrm{NS}, \mathrm{CeO}_{2} @$ PANI_NP, and $\mathrm{CeO}_{2} @$ PANI_NS samples

structures was performed. Figure 9 illustrates the photocatalyst type effect on the conversion of nitrobenzene to aniline. Photocatalytic nitrobenzene-aniline transformation with the use of $\mathrm{CeO}_{2} \mathrm{NS}$ is very small (7\%), this can be explained by the fact that $\mathrm{CeO}_{2} \mathrm{NS}$ absorbs only in the UV region while the light source used is in the visible region. Nitrobenzene-aniline transformation, photocatalytically, with the use of PANI_NP and PANI_NS was at $15 \%$ and $40 \%$, respectively. While the photocatalytic conversion with the use of $\mathrm{CeO}_{2} @ P A N I$ NP and $\mathrm{CeO}_{2} @$ PANI_NS as photocatalysts was at $70 \%$ and $100 \%$ respectively. It is clear that the addition of polyaniline nanoparticles and nanospheres increases nitrobenzene-aniline photocatalytic transformation considerably. Polyaniline nanospheres in the form of $\mathrm{CeO}_{2} @$ PANI_NS exhibit superior efficiency at about $100 \%$ conversion.

The effect of the amount of added catalyst to the reaction was also studied. Figure 10 illustrates the consequence of the dose of $\mathrm{CeO}_{2} @$
PANI_NS photocatalyst on the nitrobenzene aniline transformation. The photocatalytic transformation has been enhanced from 75 to $100 \%$ by increasing the catalyst amount from 0.3 to $0.6 \mathrm{~g} / \mathrm{L}$, respectively. The time needed for a complete nitrobenzene-aniline transformation has been reduced from 150 to 90 min by increasing the amount of $\mathrm{CeO}_{2} @$ PANI_NS from 0.6 to $1.2 \mathrm{~g} / \mathrm{L}$, respectively. This may be attributed to the improved amount of accessible active sites by the increase in the dose of the photocatalyst. If the dose was raised above $1.2 \mathrm{~g} / \mathrm{L}$ to a value of $1.5 \mathrm{~g} / \mathrm{L}$ the nitrobenzene - aniline, photocatalytically, decreases from 100 to $98 \%$ and reaction time increases from 90 to $150 \mathrm{~min}$. the higher dose of photocatalyst beyond a certain point may result in hindering penetration of light to reach all active sites of the photocatalyst.

A test on the possibility of the reuse of the catalyst was also performed. Figure 11 shows recycling and reuse of $\mathrm{CeO}_{2} @$ PANI_NS photo-catalyst on nitrobenzene-aniline transformation. The figure shows that the transformation remains constant even if the catalyst was reused for five times showing great stability of the $\mathrm{CeO}_{2} @ P A N I \_N S$ photocatalyst.

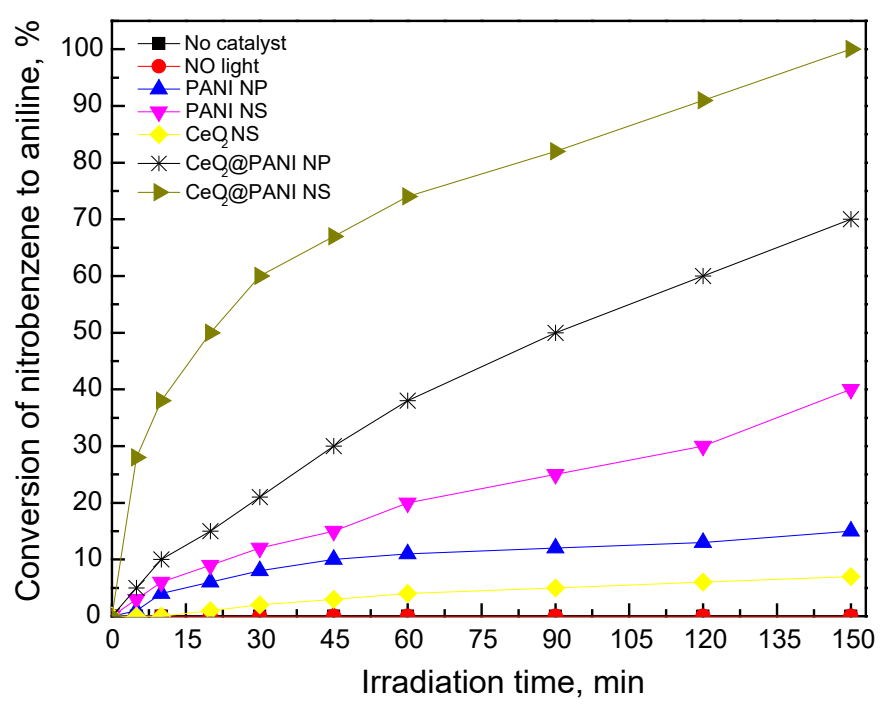

Figure 9. Effect of type of photocatalyst on the conversion of Nitrobenzene to Aniline

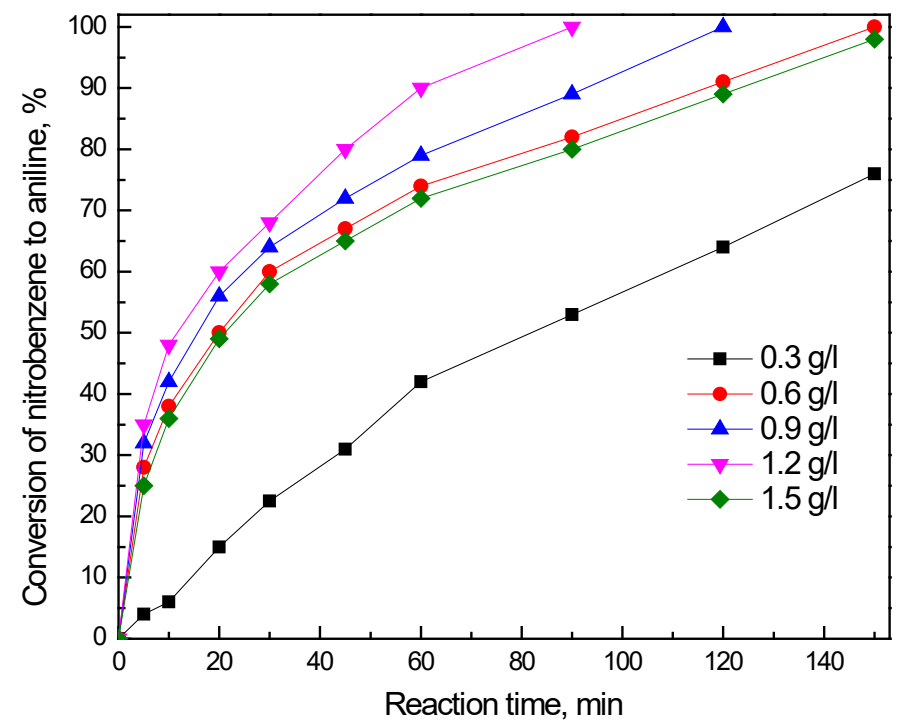

Figure 10. Effect of dose of $\mathrm{CeO}_{2} @$ PANI_NS photocatalyst on the conversion of Nitrobenzene to Aniline 


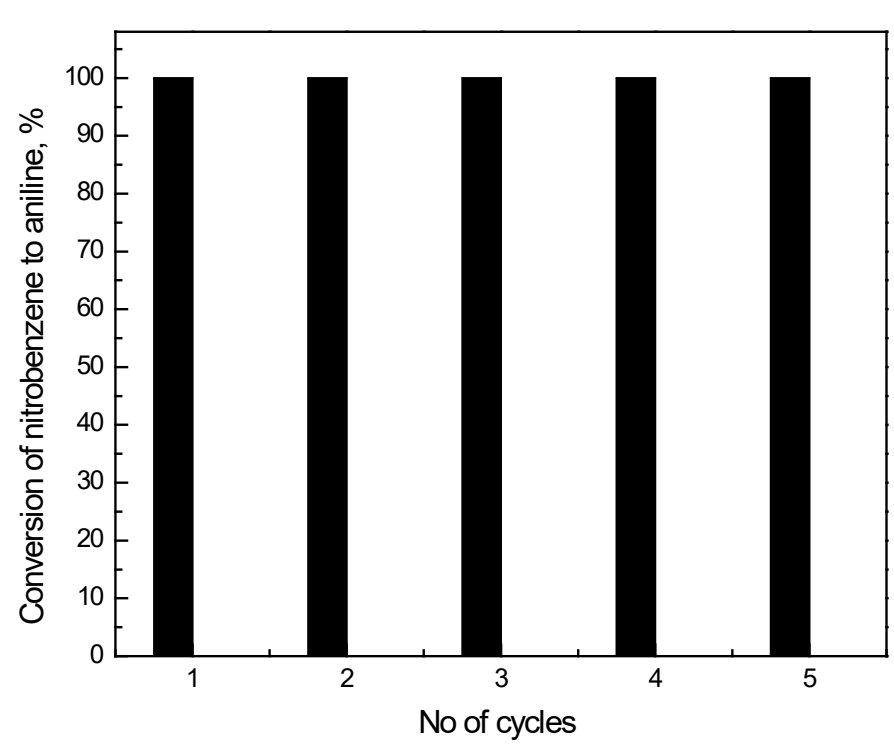

Figure 11. Recycling and reuse of $\mathrm{CeO}_{2} @$ PANI_NS photocatalyst on conversion of Nitrobenzene to Aniline

\section{Conclusion}

Polyaniline nanospheres were fabricated by the microemulsion method. Polyaniline nanoparticles were fabricated through polymerization of aniline (chemical oxidation type). $\mathrm{CeO}_{2} @ P A N I$ NS was prepared by a method that ensures the production of a uniform spherical core-shell structure. The photocatalytic efficiency of the nanocomposites was investigated by the photocatalytic reduction of nitrobenzene to aniline beneath Vis light. The values of the specific surface area of PANI_NS, CeO @PANI_NS, PANI_NP, CeO @PANI_ $\mathrm{NP}$, and $\mathrm{CeO}_{2} \_\mathrm{NS}$ are 200, 260, 40, 110 and $130 \mathrm{~m}^{2} / \mathrm{g}$, respectively, which means the NS structure has a high specific surface area. The shapes of PANI_NS (nanospheres), $\mathrm{CeO}_{2} @ P A N I \_N S$ (core-shell nanospheres), PANI_NP (nanoparticles), $\mathrm{CeO}_{2} @ P A N I \_N P$ (nanoparticles), $\mathrm{CeO}_{2}$ NS (nanospheres); were determined by TEM. The photocatalytic performance of $\mathrm{CeO}_{2} @$ PANI_NS for reduction of nitrobenzene to aniline under visible light is 1.4, 2.5, 6.6 and 14.3 times more than that of $\mathrm{CeO}_{2} @ P A N I \_N P, P A N I \_N S, P A N I \_N P$, and $\mathrm{CeO}_{2}$ NS, respectively.

\section{Acknowledgment}

This Project was funded by the Deanship of Scientific Research (DSR) at King Abdulaziz University, Jeddah, under grant no. P-05-130436. The authors, therefore, acknowledge with thanks DSR for technical and financial support.

\section{Highlights}

- A cerium oxide polyaniline nanocomposite was prepared.

- The photocatalytic properties were studied upon visible irradiation.

- A new cerium oxide polyaniline nanocomposite showed superior photocatalytic activity.
A new cerium oxide polyaniline nanocomposite remains effective and active after five cycles.

\section{References}

1. Ozbay B, Genc N, Ozbay I, Baghaki B, Zor S (2016) Photocatalytic activities of polyaniline-modified $\mathrm{TiO}_{2}$ and $\mathrm{ZnO}$ under visible light: an experimental and modeling study. Clean Techn Environ Policy 18: 2591-2601.

2. Ameen S, Akhtar MS, Kim YS, Yang OB, Shin HS (2011) An effective nanocomposite of polyaniline and $\mathrm{ZnO}$ : preparation, characterizations, and its photocatalytic activity. Colloid Polym Sci 289: 415-421.

3. Sharma S, Singh S, Khare N (2016) Synthesis of polyaniline/CdS (nanoflowers and nanorods) nanocomposites: a comparative study towards enhanced photocatalytic activity for degradation of organic dye. Colloid Polym Sci 294: 917-926.

4. Min S, Wang F, Han Y (2007) An investigation on synthesis and photocatalytic activity of polyaniline sensitized nanocrystalline $\mathrm{TiO}_{2}$ composites. J Mater Sci 42: 9966-9972.

5. Olad A, Nosrati R (2013) Preparation, characterization, and photocatalytic activity of polyaniline/ZnO nanocomposite. Res Chem Intermed 39: 3969-3979.

6. Cheng Y, An L, Zhao Z, Wang G (2014) Preparation of polyaniline/TiO composite nanotubes for photodegradation of AZO dyes. Journal of Wuhan University of Technology-Mater Sci Ed 29: 468-472.

7. Wei J, Zhang Q, Liu Y, Xiong R, Pan C, et al. (2011) Synthesis and photocatalytic activity of polyaniline- $\mathrm{TiO}_{2}$ composites with bionic nanopapilla structure. $J$ Nanopart Res 13: 3157-3165.

8. Olad A, Behboudi S, Entezami AA (2012) Preparation, characterization and photocatalytic activity of $\mathrm{TiO}_{2}$ /polyaniline core-shell nanocomposite. Bull Mater Sci 35: 801-809.

9. Agarwal S, Tyagi I, Gupta VK, Golbaz F, Golikand AN (2016) Synthesis and characteristics of polyaniline/zirconium oxide conductive nanocomposite for dye adsorption application. Journal of Molecular Liquids 218: 494-498.

10. Patil UV, Ramgir NS, Karmakar N, Bhogale A, Debnath AK, et al. (2015) Room temperature ammonia sensor based on copper nanoparticle intercalated polyaniline nanocomposite thin films. Applied Surface Science 339: 69-74.

11. Salvatierra RV, Zitzer G, Savu SA, PAlves A, Zarbin AJG, et al. (2015) Carbon nanotube/polyaniline nanocomposites: Electronic structure, doping level and morphology investigations. Synthetic Metals 203: 16-21.

12. Onn TM, Ramirez LA, Monai M, Soh T, Talati M, et al. (2016) Modification of Pd/ $\mathrm{CeO}_{2}$ catalyst by atomic layer deposition of $\mathrm{ZrO}_{2}$. Applied Catalysis B: Environmental 197: $280-285$.

13. Tang C, Sun B, Sun J, Hong X, Deng Y, et al. (2016) Solid state preparation of NiO$\mathrm{CeO}_{2}$ catalyst for NO reduction. Catal Today 281: 575-582.

14. Kim TH, Seon H, Park DW (2016) Synthesis of $\mathrm{CeO}_{2}$ nanocrystalline powders using DC non-transferred thermal plasma at atmospheric pressure. J Sol-Gel Sci Technol 27: 2012-2018.

15. He Y (2005) Synthesis of polyaniline/nano- $\mathrm{CeO}_{2}$ composite microspheres via a solidstabilized emulsion route. Materials Chemistry and Physics 92: 134-137.

16. Chuang FY, Yang SM (2008) Cerium dioxide/polyaniline core-shell nanocomposites. Journal of Colloid and Interface Science 320: 194-201.

17. Kumar E, Selvarajan P, Muthuraj D (2012) Preparation and characterization of polyaniline/cerium dioxide $\left(\mathrm{CeO}_{2}\right)$ nanocomposite via in situ polymerization. J Mater Sci 47: 7148-7156.

18. Sasikumar Y, Madhan AK, Gasem ZM, Ebenso EE (2015) Hybrid nanocomposite from aniline and $\mathrm{CeO}_{2}$ nanoparticles: Surface protective performance on mild steel in acidic environment. Applied Surface Science 330: 207-215.

19. da Silva JSM, de Souza SM, Trovati G, Sanches EA (2017) Chloride salt of conducting polyaniline synthesized in the presence of $\mathrm{CeO}_{2}$ : Structural analysis of the core-shell nanocomposite. Journal of Molecular Structure 1127: 337-344.

Copyright: (C2019 Kadi MW. This is an open-access article distributed under the terms of the Creative Commons Attribution License, which permits unrestricted use, distribution, and reproduction in any medium, provided the original author and source are credited. 